\title{
SNAP Sky Background at the North Ecliptic Pole ${ }^{1}$
}

\author{
G. Aldering \\ Institute for Nuclear and Particle Astrophysics, \\ E. O. Lawrence Berkeley National Laboratory, Berkeley, California 94720. \\ Version 1.1, Dec 31, 2001
}

\begin{abstract}
I summarize the extant direct and indirect data on the sky background SNAP will see at the North Ecliptic Pole over the wavelength range $0.4<\lambda<1.7 \mu \mathrm{m}$. At the spatial resolution of SNAP the sky background due to stars and galaxies is resolved, so the only source considered is zodiacal light. Several models are explored to provide interpolation in wavelength between the broadband data from HST and COBE observations. I believe the input data are now established well enough that the accuracy of the sky background presented here is sufficient for SNAP simulations, and that it will stand up to scrutiny by reviewers.
\end{abstract}

\section{The Nature of the Background}

The SNAP supernovae program will concentrate on fields towards the north ecliptic pole (NEP), and possibly the south ecliptic pole. Since the SNAP imager will operate in the sky background-limited regime the exposure times needed to reach a given $S / N$ will be proportional to the sky background. Thus, determination of the expected background level is essential for estimating the SNAP exposure time budget.

The principal background for $0.4<\lambda<1.7 \mu \mathrm{m}$ will be from zodiacal light — solar light reflected from dust in the inner solar system. Stars and galaxies will be resolved by SNAP so their contribution to the diffuse background will come from scattering of their light within the telescope — which we hope will be negligable for SNAP. In its high-earth orbit SNAP will only suffer from scattered light due to the Earth for limited intervals, unlike HST which has only about $1 / 3$ of its orbit free of serious Earthshine contamination (STIS Zodiacal Webpage 2001). Given the limited Earthshine contribution for SNAP, it has not been treated here. HST also experiences elevated background whenever it is not in Earth shadow — this may be due to unbaffled sunlight which is scattered into the telescope. This is a potentially serious issue which SNAP will have to guard against. Since it is not a quantifiably irreducible background, it is not treated here.

The IRAS map shown in Fig. 1 shows that the NEP is covered by Galactic dust, so SNAP will be forced to select a field at lower ecliptic latitude $\left(\beta \sim 75^{\circ}\right)$ to avoid Galactic extinction. This will also eliminate diffuse starlight scattered by the Galactic dust. Therefore, the zodiacal light background will be modulated annually as the angle between the SNAP field and the Sun varies from $75^{\circ}$ to $105^{\circ}$. The scattering phase function of the solar system dust is such that this modulation is only about $\pm 15 \%$. A slight additional annual modulation (about $\pm 4 \%$ ) will occur due to the tilt of Earth's orbit relative to the dust cloud responsible for the zodical light. These effects are small, so the distinction between the true SNAP field and the NEP will be ignored here.

\section{Zodiacal Background}

Chapter 8 of Leinert et al. (1998) discusses the properties of the zodical background in detail. The general findings of Leinert et al. (1998) are still valid and so will not be repeated here. However, several new data

${ }^{1}$ Reference as: 'Aldering, G. 2001, LBNL report number LBNL-51157' 
North Ecliptic/Galactic Pole Dust Map

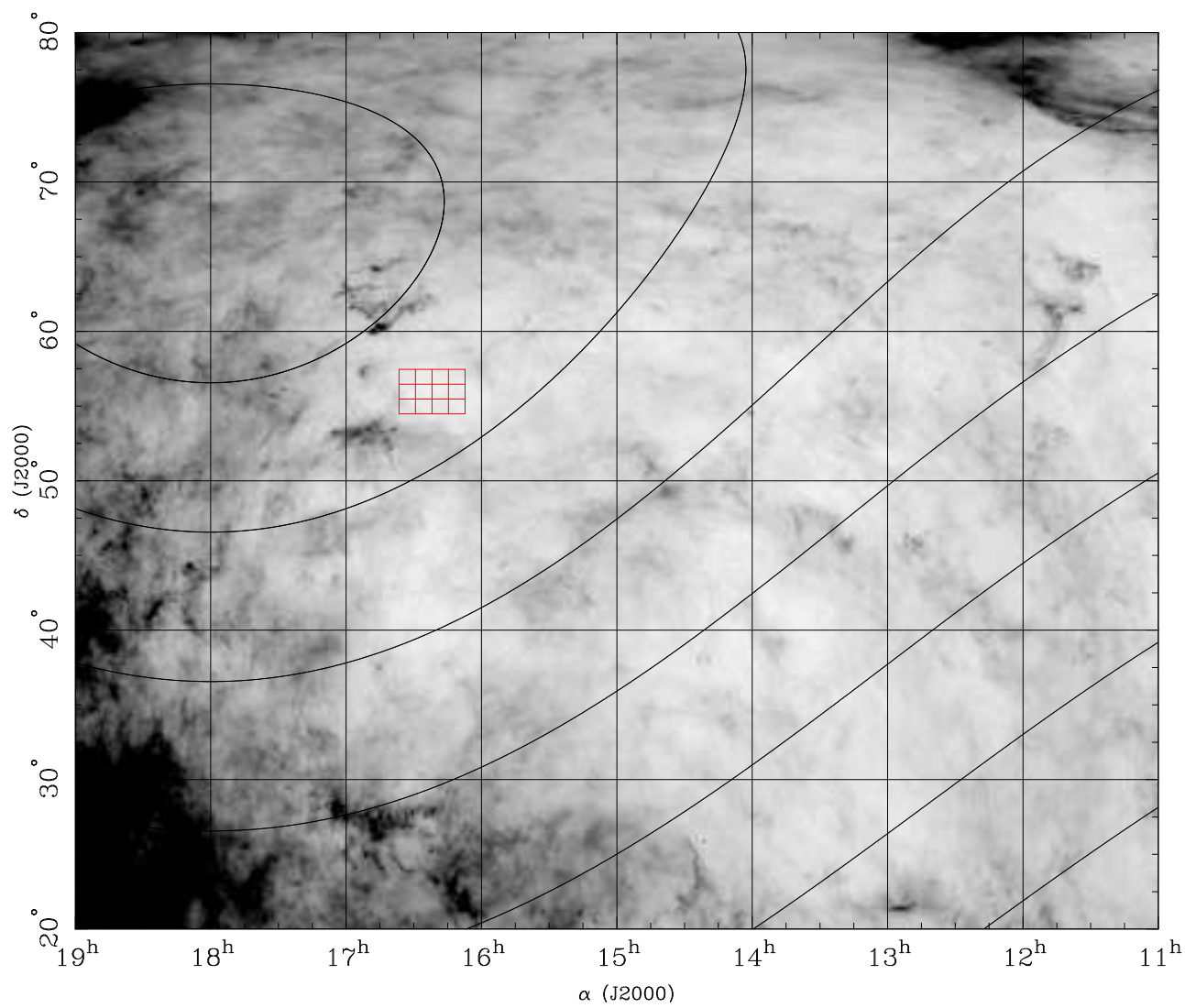

Figure 1: Dust map in low-dust region near NEP, based on Schlegel, Finkbiener, and Davis (1998). The curves indicate lines of constant ecliptic latitude, starting at $+80^{\circ}$ in the upper left. A low dust region suitable for SNAP is indicated

sources not considered in Leinert et al. (1998) are now available, and appear to resolve several outstanding issues.

Table 17 of Leinert et al. (1998) reports a NEP surface brightness of $1.81 \times 10^{-18} \mathrm{erg} \mathrm{cm}^{-2} \mathrm{~s}^{-1} \AA^{-1} \operatorname{arcsec}^{-2}$ at $\lambda=0.50 \mu \mathrm{m}$; this corresponds to a V-band surface brightness of $23.3 \mathrm{mag} / \mathrm{arcsec}^{2}$. According to Leinert et al. (1998) the zodiacal light is redder than the solar spectrum, but the amount of reddening is dependent on solar elongation. For an elongation of $90^{\circ}$ the zodiacal spectrum normalized to the value at $\lambda=0.50 \mu \mathrm{m}$ behaves as:

$$
\frac{f(\lambda)}{f(0.50 \mu \mathrm{m})}=1.0+0.90 \ln \left(\frac{\lambda}{0.50 \mu \mathrm{m}}\right) \text { for } \lambda<0.50 \mu \mathrm{m}
$$

and

$$
\frac{f(\lambda)}{f(0.50 \mu \mathrm{m})}=1.0+0.60 \ln \left(\frac{\lambda}{0.50 \mu \mathrm{m}}\right) \text { for } 0.50<\lambda<2.50 \mu \mathrm{m}
$$




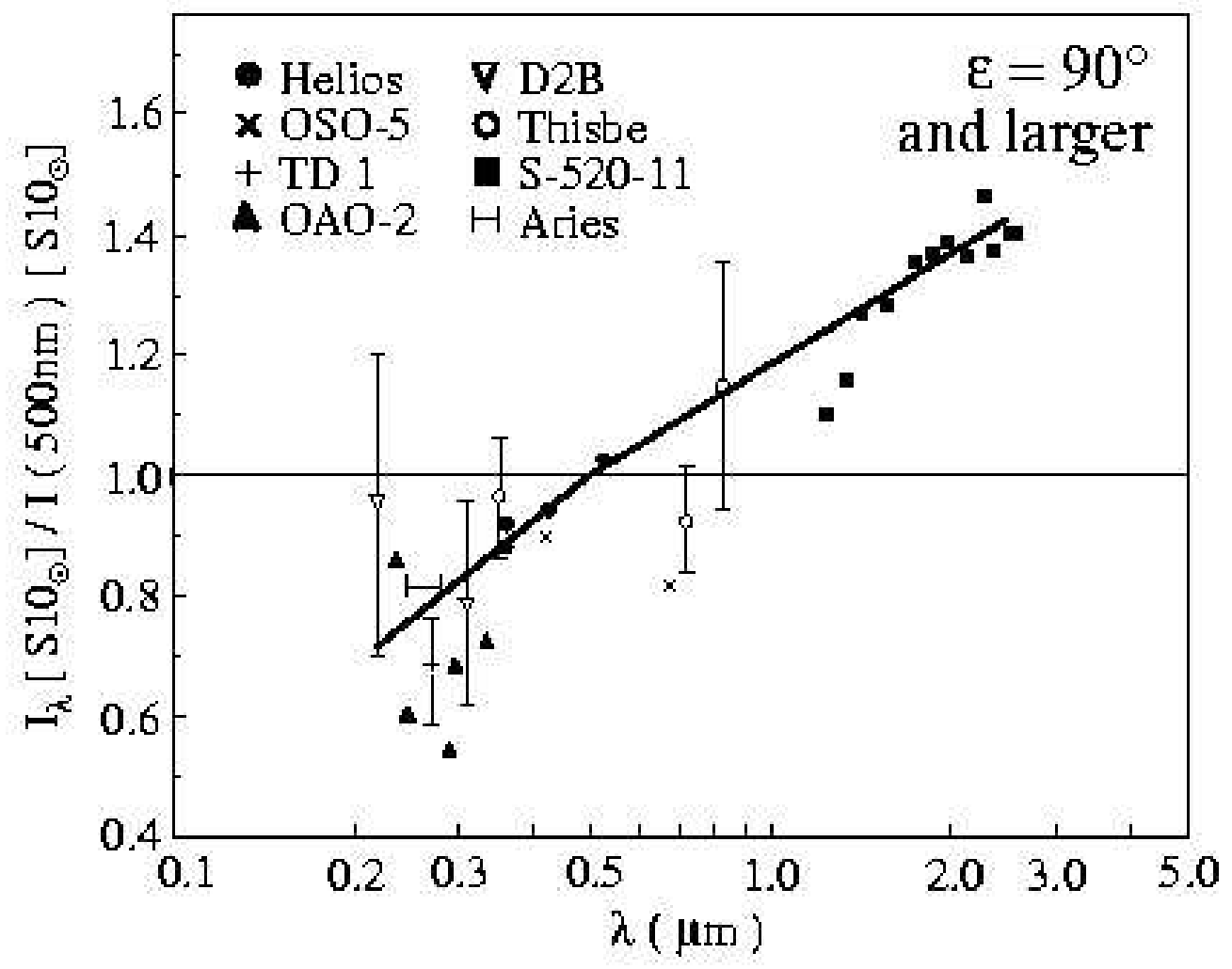

Figure 2: Zodiacal background color data normalized to the solar spectrum for solar elongation of $90^{\circ}$ or greater. The broken solid line is the Leinert reddening prescription.

These formulae are from observations of regions of the sky not at the NEP, but should apply if the dust scattering function is a unique function of scattering angle since it does include regions with solar elongations of $\sim 90^{\circ}$ such as SNAP will experience. The data used by Leinert et al. (1998), and the resulting broken linear fits given above, are shown in Fig. 2. Note that the NIR sky background measurements in Fig. 2 (see Matsuura et al. (1994)) include a small $(\sim 15 \%)$ contribution from stars and galaxies, which SNAP will resolve. This means that the reddening in Fig. 2 has likely been slightly overestimated at NIR wavelengths. A solar spectrum (Colina, Bohlin, \& Castelli 1996) using the Leinert et al. (1998) NEP normalization and reddening formula is shown in Fig. 3.

\subsection{HST Hubble Deep Field - North}

In order to confirm the Leinert et al. (1998) specification for the zodiacal background several other measurements were examined. The first of these is my determination of dark-sky values obtained by the HST during the observations for the northern Hubble Deep Field (HDF-N; Williams et al. (1996)) using values listed at the HDF Webpage (2001). Since the sky background varied during the course of the HDF-N observations due to contamination from Earthshine and solar scattered light, the locus of minimum values was taken as the zodiacal background level. The HDF-N is at $\beta \sim 57^{\circ}$, and the observations were obtained at 


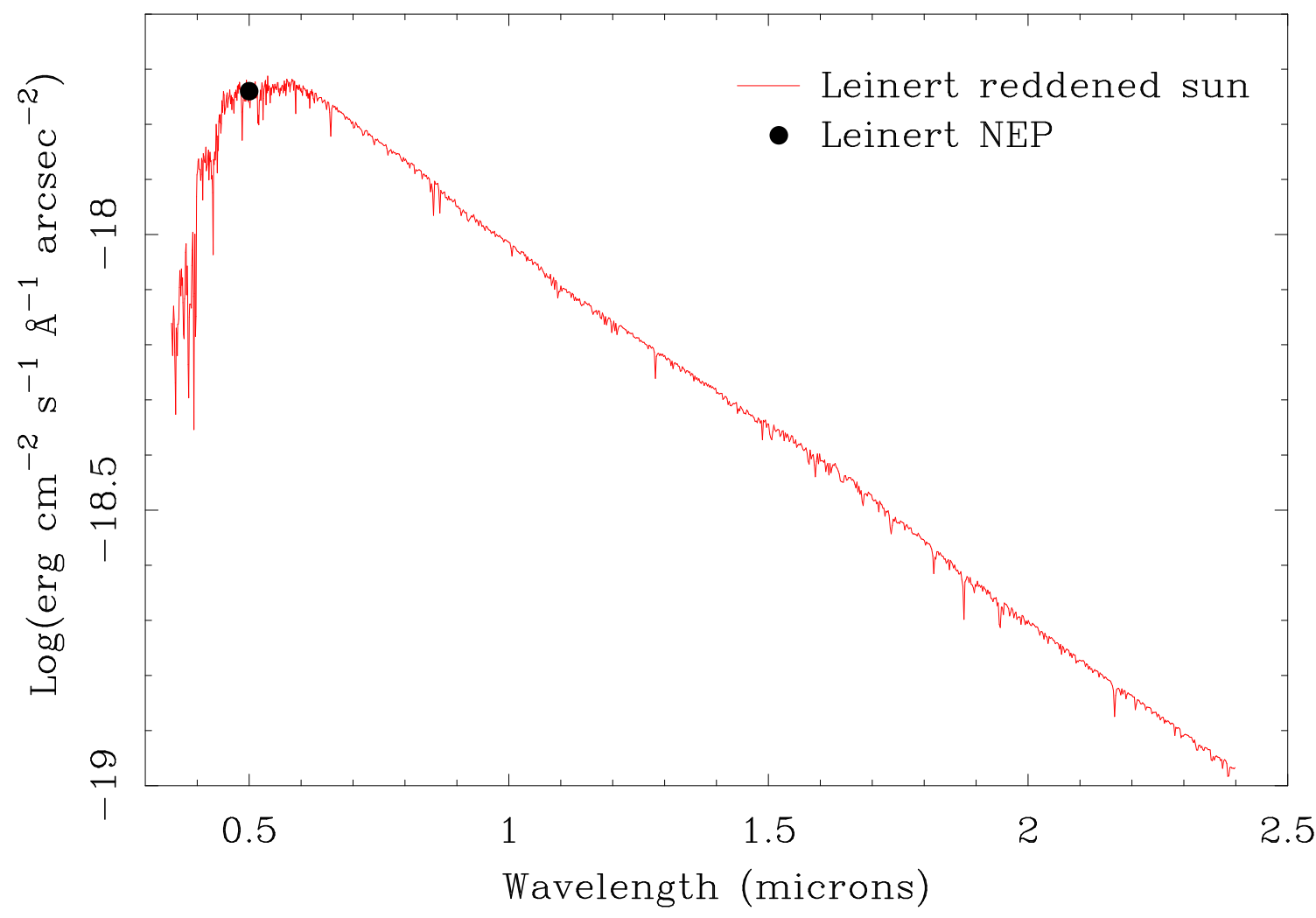

Figure 3: Solar spectrum reddened according to Leinert prescription, and normalized to a $V$-band surface brightness of $23.3 \mathrm{mag} \operatorname{arcsec}^{-2}$.

$\lambda-\lambda_{\odot} \sim 90^{\circ}$. Therefore, the raw HDF-N sky meaurements must be decreased by $12 \%$ (see Table 16 of Leinert et al. (1998)) to give the values they would have at the NEP ${ }^{2}$. The raw and corrected values are listed in Table 1, and corrected values are overlaid on the Leinert-reddened solar spectrum in Fig. 4. The HST sky values are all about 3\% fainter than the Leinert-normalized zodiacal background, but this is consistent with the $5 \%$ Leinert normalization uncertainty and the $2 \%$ broadband filter calibration uncertainties.

\subsection{COBE Diffuse Infrared Background Experiment}

The Diffuse Infrared Background Experiment (DIRBE; Boggess et al. (1992)) on the Cosmic Background Explorer (COBE) observed the entire sky at wavelengths $-1.25 \mu \mathrm{m}$ and $2.20 \mu \mathrm{m}$ - which span NIR wavelengths where SNAP will observe. DIRBE had low angular resolution so its signal includes contributions from the interstellar medium, stars and galaxies which SNAP will resolve. SNAP is also likely to resolve the cosmic infrared background which comprises part of the DIRBE signal since this signal most likely comes from galaxies. Gorjian, Wright, \& Chary (2000) determined the DIRBE background at $2.2 \mu \mathrm{m}$ after correcting for foreground stars in a dark region of the DIRBE map at $\beta \sim+51$. They used a model of the

\footnotetext{
${ }^{2}$ Note that in earlier work circulated this summer I used HST sky values which had not been adjusted to the NEP. Therefore, the values used here are slightly fainter than previous values.
} 


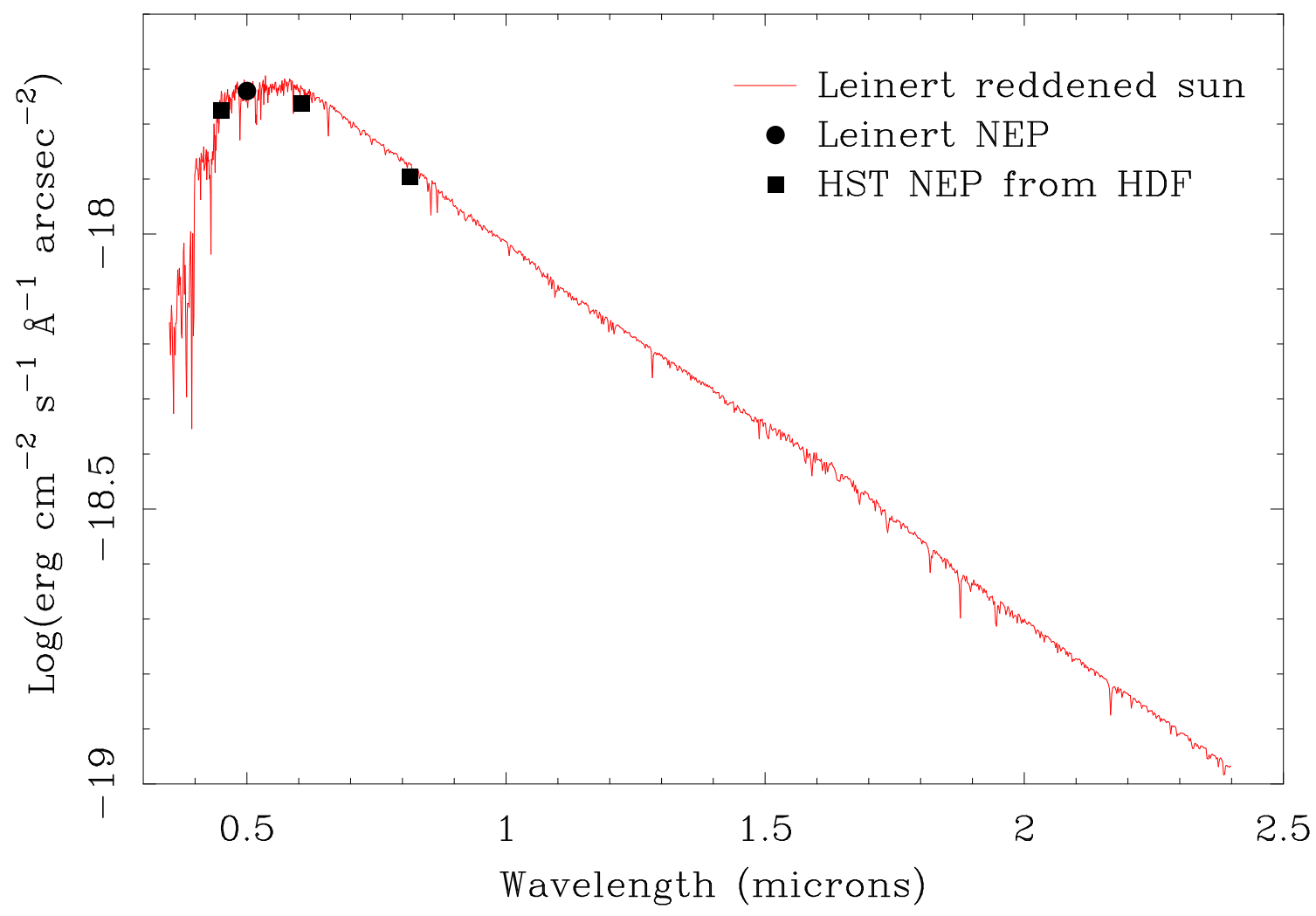

Figure 4: The same as Figure 3, but now with Hubble Deep Field minimum sky background points overlaid.

zodiacal light that is claimed to be superior to that used previously (e.g. Kelsall et al. (1998)) at $2.2 \mu \mathrm{m}$. Note that at this wavelength about $74 \%$ of the DIRBE signal from this field is due to zodiacal light, so errors in the contamination corrections amount to only a few percent. A factor of 0.75 reduction in the Gorjian, Wright, \& Chary (2000) zodiacal background value of $1.7 \times 10^{-19} \mathrm{erg} \mathrm{cm}^{-2} \mathrm{~s}^{-1} \AA^{-1} \operatorname{arcsec}^{-2}$ is needed to correct to the equivalent NEP background level.

In addition, Wright (2001a) has compared the DIRBE data with various zodiacal light models in the direction of the Lockman Hole $\left(\beta \sim+46^{\circ}\right)$. This region has very low $\mathrm{HI}$ column density, and so there is very little contribution from the ISM. The model values range from $0.14-0.18 \mathrm{MJy} / \mathrm{sr}$ at $1.25 \mu \mathrm{m}$ and $0.09-0.12$ $\mathrm{MJy} / \mathrm{sr}$ at $2.2 \mu \mathrm{m}$. In Table 2 the mean and standard deviations of the model estimates is given at each DIRBE 
Table 1: Raw and NEP-corrected sky background from the HDF-N image set

\begin{tabular}{|c|c|c|c|}
\hline Filter & $\begin{array}{c}\text { Wavelength } \\
\text { microns }\end{array}$ & $\begin{array}{l}\text { Raw Counts } \\
\text { DN/pix/sec }\end{array}$ & $\begin{array}{c}\operatorname{Raw~f}_{\lambda} \operatorname{NEP~f}_{\lambda} \\
\mathrm{erg} / \mathrm{cm}^{2} / \mathrm{s} / \AA ̊ \operatorname{arcsec}^{2}\end{array}$ \\
\hline F450W & 0.450 & 0.00210 & $1.87 \mathrm{e}-18 \quad 1.67 \mathrm{e}-18$ \\
\hline F606W & 0.606 & 0.01024 & $1.93 \mathrm{e}-18$ \\
\hline F814W & 0.814 & 0.00567 & $1.42 \mathrm{e}-18$ \\
\hline
\end{tabular}

Table 2: Raw and NEP-corrected sky background from DIRBE

\begin{tabular}{|ccccc|}
\hline $\begin{array}{c}\text { Wavelength } \\
\text { microns }\end{array}$ & $\begin{array}{c}\text { Raw f }_{\lambda} \\
\mathrm{erg} / \mathrm{cm}^{2} / \mathrm{s} / \AA \mathrm{A}_{\lambda} / \operatorname{arcsec}^{2}\end{array}$ & $\mathrm{NEP} \mathrm{f}_{\lambda}$ & Source \\
\hline 2.20 & $1.67 \mathrm{e}-19$ & $0.06 \mathrm{e}-19$ & $1.26 \mathrm{e}-19$ & Gorjian 2000 \\
1.25 & $7.26 \mathrm{e}-19$ & $0.83 \mathrm{e}-19$ & $5.37 \mathrm{e}-19$ & Wright 2001 \\
2.20 & $1.62 \mathrm{e}-19$ & $0.18 \mathrm{e}-19$ & $1.20 \mathrm{e}-19$ & Wright 2001 \\
\hline
\end{tabular}

band of interest ${ }^{3}$, and then corrected to the NEP assuming the latitude dependence of Leinert et al. $(1998)^{4}$.

\subsection{Rocket \& Balloon Flights}

Over the years numerous rocket and balloon flights have measured the total NIR sky brightness with low angular resolution in small fields (Matsuura et al. 1994; Mandolesi et al. 1998). However, in all cases the pure zodiacal component cannot be determined; the annual variation of the zodiacal background used to isolate this component from stars, galaxies, the interstellar medium, and the cosmic IR background with DIRBE cannot be exploited due to the limited duration of these rocket and balloon experiments. However, these flights have found good agreement with the total DIRBE measurements. Fig. 17 of Matsuura et al. (1994) is useful in establishing that the NIR background varies smoothly and monotonically between the $1.25 \mu \mathrm{m}$ and $2.2 \mu \mathrm{m}$ DIRBE bands.

\section{Comparison with Other Sources}

SNAP originally used a sky background consisting of values presented on the HST STIS webpage (STIS Earthshine Webpage 2001) in the optical and values measured from a Ball Aerospace sky background plot in the NIR. This spectrum had a discontinuity between the optical and the NIR. Mike Lampton raised some questions about this sky background spectrum. Re-examination of the STIS values indicate that they are simply the solar spectrum, normalized to a V-band surface brightness of $23.3 \mathrm{mag} / \mathrm{arcsec}^{2}$, with no account

\footnotetext{
${ }^{3}$ Note that in previous work distributed this past summer I had selected the brightest of the model values, rather than the mean, so the NIR zodiacal sky values used here are slightly fainter.

${ }^{4}$ Since COBE scanned great circles roughly perpendicular to the Sun (Smoot, priv. comm.; Boggess et al. (1992)), the Lockman Hole would have been located at $\lambda-\lambda \odot \sim 90^{\circ}$ and $\beta+46$ at the time it was observed by DIRBE. In addition, the zodiacal light at $1.25 \mu \mathrm{m}$ and $2.2 \mu \mathrm{m}$ is scattered, not re-radiated. Thus, Table 16 of Leinert et al. (1998) can be used to calculate that the Lockman Hole zodiacal levels must be multiplied by a factor of 0.74 to give the equivalent NEP values. This scaling is confirmed by the model uncertainties given in Wright and Johnson (2001) which are described as being 5\% of the NEP zodiacal light, and which therefore can be used to infer the NEP zodiacal light values
} 


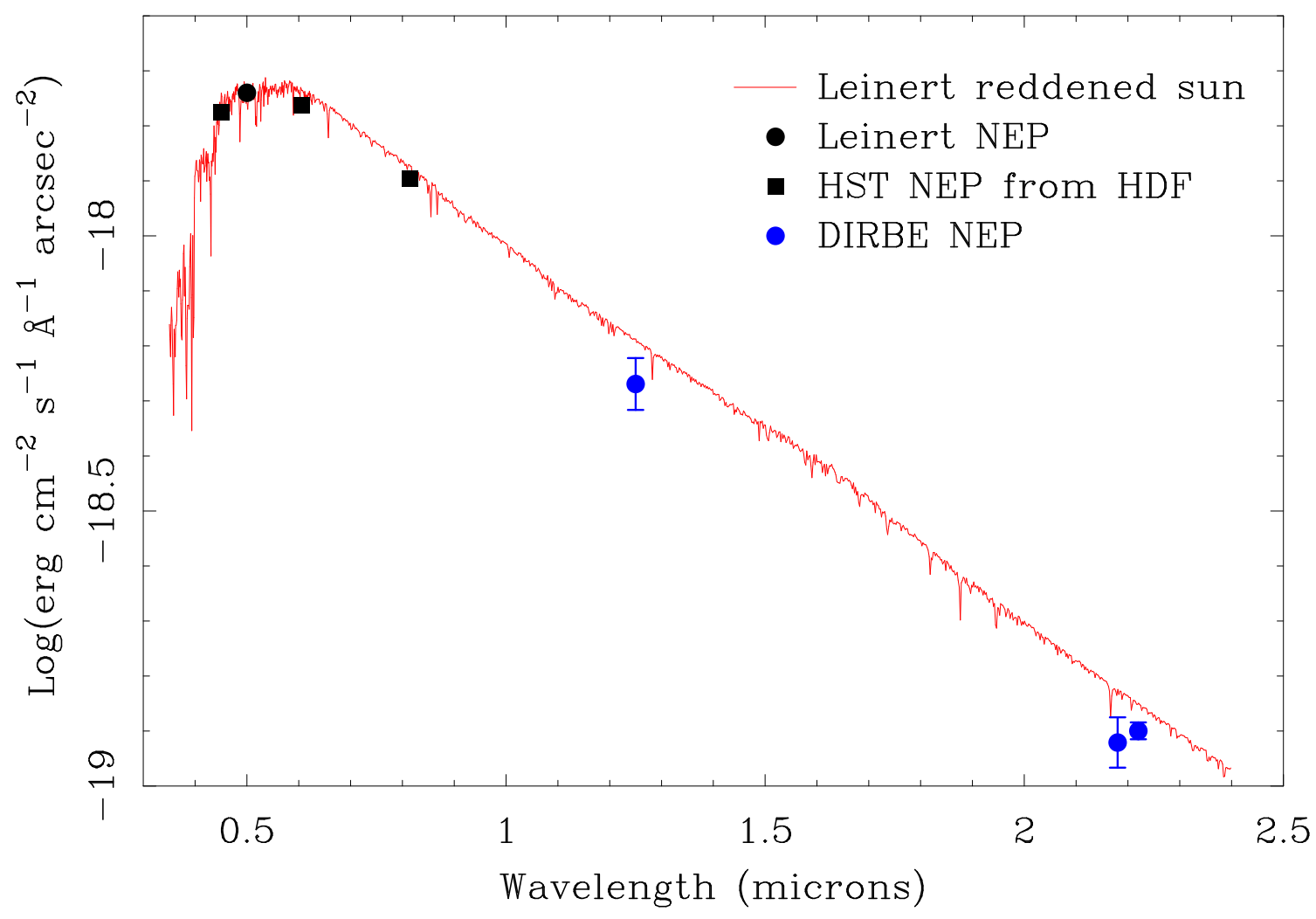

Figure 5: The same as Figure 4, but now with DIRBE sky background points overlaid.

for reddening like that shown by Leinert et al. (1998). None of the HST webpages justify this choice for the background. Subsequent discussions with Ralph Bohlin and Kailash Sahu of HST led to their adoption of a zodiacal background very similar to that presented in this memo.

Jay Frogel and I examined the background levels given by the NGST exposure time calculator (ETC; NGST ETC Webpage (2001)). The NGST zodiacal light is based on the model of Wright (1998), and is specific to the location of NGST at L2 due to asymmetries in the dust distribution. Since the COBE data used in Wright (1998) begin at $1.25 \mu \mathrm{m}$ the NGST exposure time calculator extrapolates the background level for shorter wavelengths, which therefore are suspect. Use of the NGST background values required that we take the NGST counts and convert them to surface brightness using the NGST efficiencies listed in the instrument tables; in some cases this procedure was approximate since the ETC interpolates between table values while we used efficiencies tabluated at the nearest appropriate wavelength.

In Fig. 6 the NGST background levels are overlaid on the previously discussed data. The NGST values are systematically above the measurements that I have derived, with a mean offset of about 0.1 dex (25\%). This is a bit surprising since the values in Table 2 come from a model which is only slightly newer and more refined than that used by NGST. Roughly half of the offset could be explained if NGST chose a model which is one of the brightest models among the seven Lockman Hole models of Wright (2001a). It is also possible that NGST has chosen to not subtract the cosmic IR background, whose brightness is about $15 \%$ of the NEP 


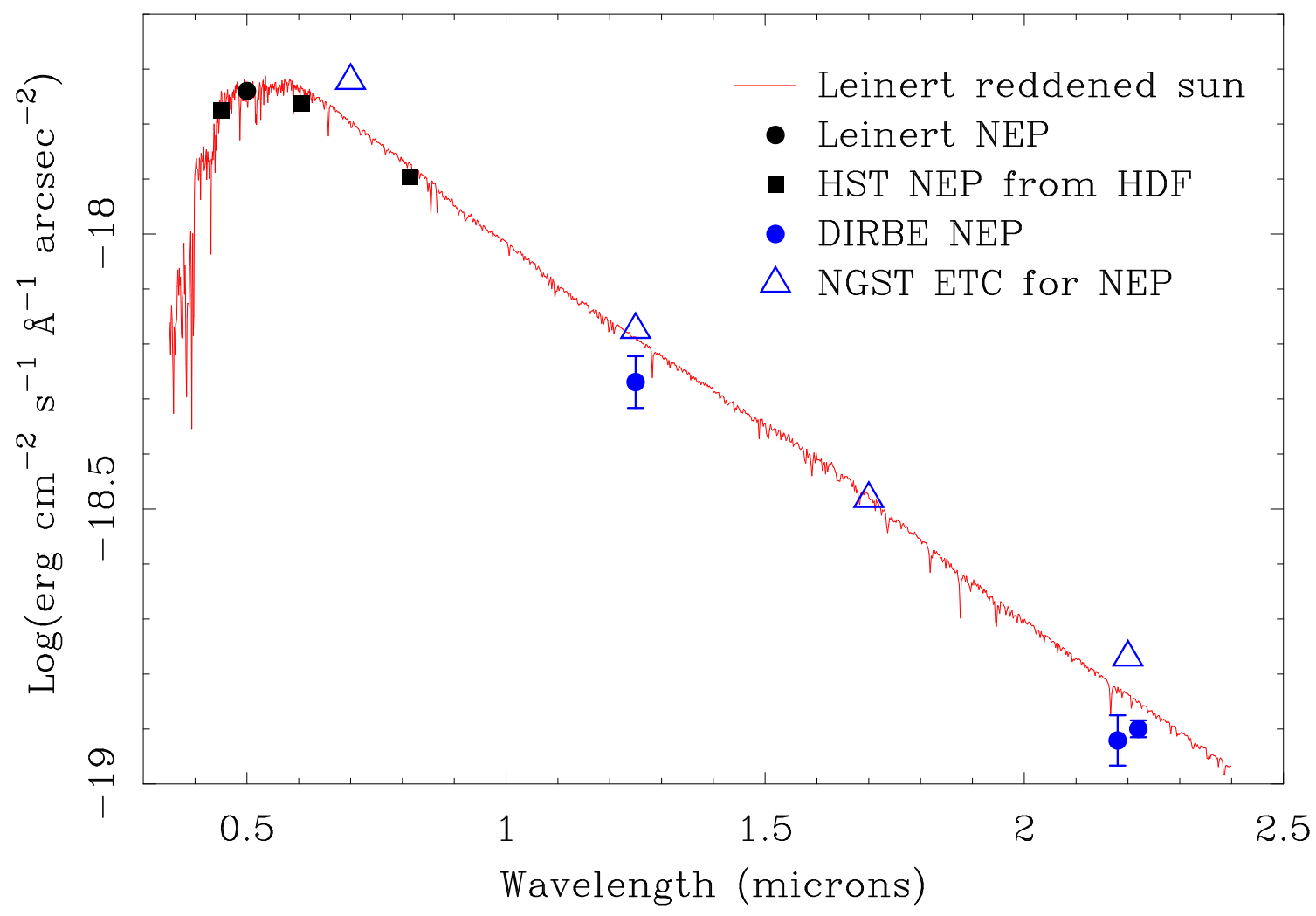

Figure 6: The same as Figure 5, but now with NGST ETC sky background points overlaid.

zodiacal component. At $1.25 \mu \mathrm{m}$ and $1.7 \mu \mathrm{m}$ the NGST values agree well with the Leinert et al. (1998) normalized reddened solar spectrum, but they are too bright at $0.7 \mu \mathrm{m}$ and $2.2 \mu \mathrm{m}$.

Overall there is good agreement from all reliable sources at optical wavelengths, but some disagreement in the NIR. Therefore, some choices must be made to come up with a final recommendation for the zodiacal background SNAP should use. First, given our experience that backgrounds obtained from HST webpages have not been well-supported with direct measurements, I recommend that we consider the NGST comparison presented above as suggestive of agreement with the values in Table 2, rather than indicative of disagreement. Further, given that the S-520-11 rocket data used in the NIR to determine the Leinert et al. (1998) reddening relation includes not just the zodiacal light but also stars and galaxies, I don't think the disagreement of the Leinert et al. (1998) normalized reddened solar spectrum with the NIR values in Table 2 is very serious. Therefore, I will proceed to find a good description of zodiacal background based on the data as given in Tables 1 and 2.

\section{Synthesis}

The data in Table 2 - and even the F814W value in Table 1 - indicate that the zodiacal background is not quite as red as inferred by Leinert et al. (1998). This is in line with expectations since the NIR data used 
by Leinert et al. (1998) contained non-zodiacal light. I have found that lowering the overall normalization by 0.01 dex and changing the solar spectrum reddening coefficient from 0.60 to 0.48 (see the Leinert reddening equations given above) gives excellent agreement with the data I have assembled. This is shown in Fig. 7.

The above reddened solar spectrum can be approximated by a $5800^{\circ} \mathrm{K}$ blackbody multiplied by $\lambda^{0.36}$ :

$$
f(\lambda)=10^{-17.00} \lambda^{-4.64}\left(\exp \left[\frac{h c}{k 5800 \lambda}\right]-1\right)^{-1} \operatorname{erg~cm}{ }^{-2} \mathrm{~s}^{-1} \AA^{-1} \operatorname{arcsec}^{-2}
$$

This approximation is overlaid in Fig. 7. The advantage of this approximation is that it is easy to calculate - it is continuous and doesn't require having a solar spectrum on hand — unlike the Leinert et al. (1998) formulation. However, note that between 1.25 and $2.2 \mu \mathrm{m}$ the blackbody is too faint; the rocket data of Matsuura et al. (1994) behaves like the reddened solar spectrum, so this blackbody approximation would be about $15 \%$ too faint at SNAP's reddest wavelength of $1.7 \mu \mathrm{m}$.

Alternatively, a broken log-linear relation is a useful approximation which better tracks the reddened solar spectrum beyond $0.6 \mu \mathrm{m}$.:

$$
\log _{10}(f(\lambda))=-17.755 \text { in } \operatorname{erg~cm}^{-2} \mathrm{~s}^{-1} \AA^{-1} \operatorname{arcsec}^{-2} \text { for } 0.40<\lambda<0.61
$$

and

$$
\log _{10}(f(\lambda))=-17.755-0.730(\lambda-0.61 \mu \mathrm{m}) \text { in } \mathrm{erg} \mathrm{cm}^{-2} \mathrm{~s}^{-1} \AA^{-1} \operatorname{arcsec}^{-2} \text { for } 0.61<\lambda<2.20
$$

This parameterization is a very good match to the data and tracks the reddened solar spectrum quite well. The largest deviation is at the blue end; here $\mathrm{SNe}$ will be so bright that the background level will not influence the exposure time. The above parameterization is $14 \%$ fainter in the optical than the similar zodiacal light broken power parameterization I suggested this summer, and it is not quite as red in the NIR as that parameterization. As originally stated, the values used in that previous parameterization catered more to the background values given by the NGST ETC.

\section{Conclusion}

A dataset of zodiacal background levels has been assembled which is well described physically as arising from sunlight scatterd off dust particles having a red albedo (typical of most solar system bodies). The optical data are direct measurements, so are unlikely to change significantly. The values in the NIR are based on modeling of direct measurements which have modest contamination from sources that will be resolved by SNAP and thus not contribute to SNAP's background. These NIR values are unlikely to change by more than 10-20\% once high-resolution observations (e.g., from SIRTF) are available. Therefore, I think that the input data are sufficiently robust for SNAP simulation purposes.

Parameterizations in terms of a) a reddened solar spectrum, b) a reddened blackbody, and c) a broken power law are all good to excellent representations of the data. The broken power law seems to offer the best compromise between ease of calculation and accuracy, and is recommended as the baseline for simulations. (However, since I've heard a variety of opinions on this, I want to make clear that I think the other two parameterizations are good and also could be used.)

\section{Acknowledgements}

This work was instigated by Mike Lampton's persistent questioning of the previous SNAP zodiacal background model. I thank Mike, as well as Jay Frogel, Andy Fruchter, and Ralph Bohlin for helpful discussions 


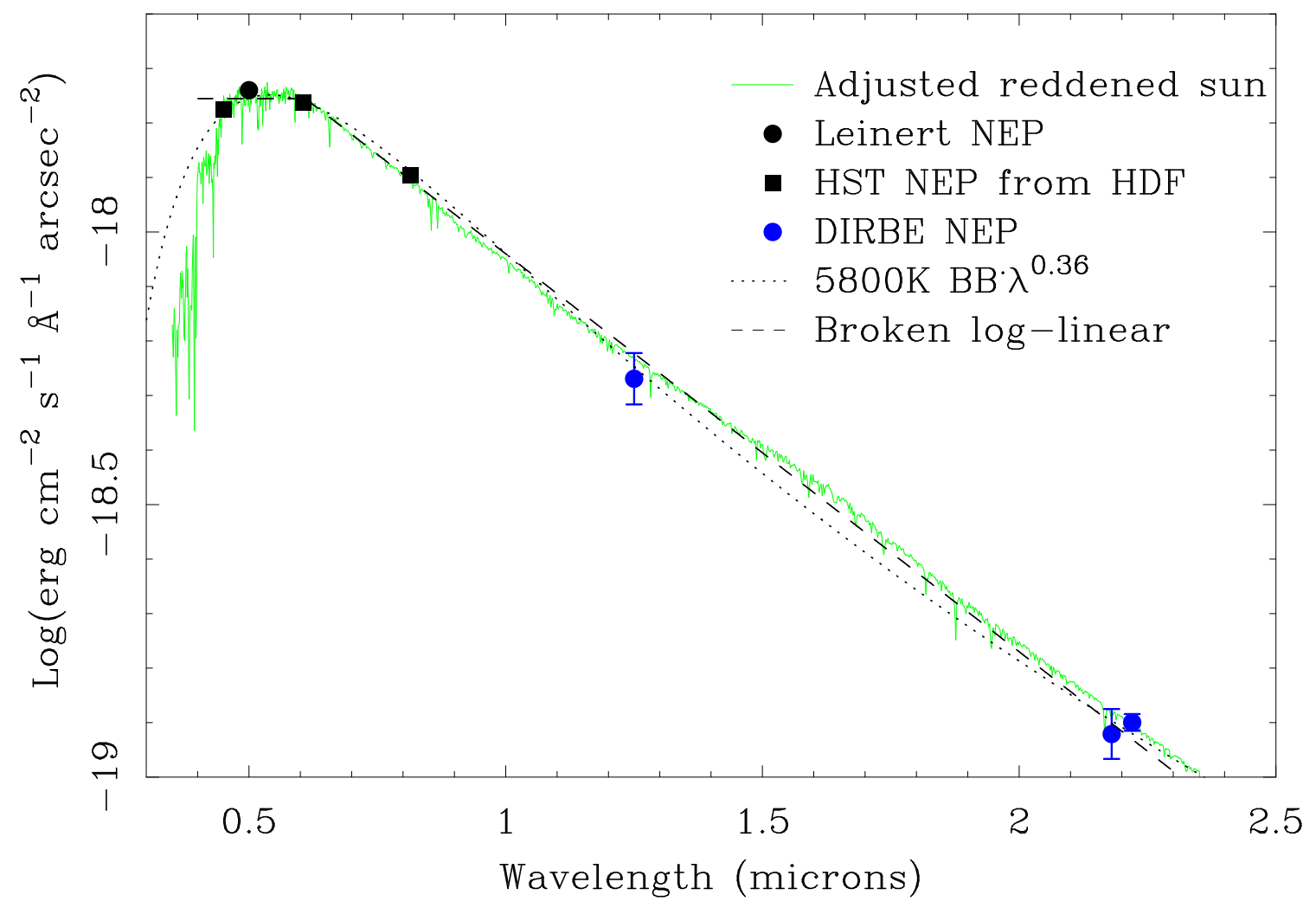

Figure 7: The solar spectrum, adjusted to match the observed zodiacal background (solid green). Simplified characterization - a $5800^{\circ} \mathrm{K}$ blackbody scaled by $\lambda^{0.36}$ (dotted black). Broken power-law parameterization (dashed black).

and assistance.

\section{References}

Boggess, N. W. et al. 1992, Ap. J., 397, 420.

Colina, L., Bohlin, R. C., \& Castelli, F. 1996, A. J., 112, 307.

HDF Webpage: http://www.stsci.edu/ftp/science/hdf/logs/MASTERLOG.

Finkbeiner, D. P., Davis, M., \& Schlegel, D. J. 1999, Ap. J., 524, 867.

Gorjian, V., Wright, E. L., \& Chary, R. R. 2000, Ap. J., 536, 550.

Kelsall, T. et al. 1998, Ap. J., 508, 44.

Leinert, C. et al. 1998, A\&ApS, 127, 1. 
Matsuura, S., Kawada, M., Matsuhara, H., Matsumoto, T., Noda, M., Tanaka, M., \& Bock, J. J. 1994, P. A. S. P., 106, 770.

Mandolesi, N. et al. 1998, A\&A, 331, 463.

NGST ETC Webpage: http://www.ngst.stsci.edu/nms/main/nms_etc_form.html.

Schlegel, D. J., Finkbeiner, D. P., \& Davis, M. 1998, Ap. J., 500, 525.

STIS Earthshine plot: www.stsci.edu/instruments/stis/documents/handbooks/cycle11/c06exptime6.html\#337664.

STIS Background Table: http://www.stsci.edu/instruments/stis/documents/handbooks/cycle11/c06_exptime9.html.

Williams, R. E. et al. 1996, A. J., 112, 1335.

Wright, E. L. 2001, Ap. J., 496, 1.

Wright, E. L. 2001, IAU Symposium 204, "The Extragalactic Infrared Background and its Cosmological Implications," ed. Martin Harwitt \& Michael Hauser (astro-ph/0106412).

Wright, E. L. and Johnson, B. D. 2001, astro-ph/0107205. 